\title{
Laboratory parameters can be used to differentiate renal infarction and urolithiasis in patients who are admitted to the emergency department with flank pain
}

\author{
(DHasan Basri Çetinkaya, @Tufan Alatlı \\ Balıkesir Universty Faculty of Medicine, Department of Emergency Medicine, Balıkesir,Turkey
}

Cite this article as: Çetinkaya HB, Alatlı T. Laboratory parameters can be used to differentiate renal infarction and urolithiasis in patients who are admitted to the emergency department with flank pain. J Health Sci Med 2021; 4(4): 462-465.

\begin{abstract}
Aim: We aimed to determine the predictive value of laboratory parameters for the distinction between urolithiasis (UL) and renal infarction (RI) in patients presenting to the emergency department with flank pain complaint.

Material and Method: In our retrospective study, the files of 73 patients who presented to the emergency department (ED) with flank pain and whose costovertebral angle tenderness was positive were reviewed. Routine blood tests and the results of contrast-enhanced abdominal computed tomography were obtained. The patients were divided into two groups as RI and UL according to the results of computed tomography. Accordingly, 8 patients were found to have RI, and 65 had UL.

Results: Lactate dehydrogenase (LDH) and platelet lymphocyte ratio (PLR) values were significantly higher in the RI group compared to the UL group ( $\mathrm{p}<0.001-\mathrm{p}=0.045)$. In ROC curve analysis, the AUC values of LDH and PLR were determined as LDH (AUC=0.983, $\mathrm{p}<0.001)$ and PLR ( $\mathrm{AUC}=0.719, \mathrm{p}=0.015)$ for their diagnostic performance in distinguishing between RI and UL.

Conclusion: We think that the use of LDH level and PLR value may be guiding in making a distinction between UL, one of the frequent reasons for presenting to ED, and RI, in which rare but early diagnosis of plank pain is critical in terms of renal parenchymal damage.
\end{abstract}

Keywords: Emergency department, flank pain, urolithiasis, renal infarction

\section{INTRODUCTION}

Flank pain is one of the important reasons for presenting to the emergency department (ED). Although many diseases, such as pyelonephritis, urinary system diseases such as ureteropelvic junction obstruction, or intestinal, gynecological, and retroperitoneal diseases can present with similar clinics, most of them turn out to be urolithiasis (UL) cases (71.4\%) (1). Even though it is rare (0.004-0.007\%), one of the most important etiology is renal infarction (RI). RI is permanent renal parenchymal damage characterized by impaired blood flow to the kidney as a result of renal artery occlusion. It has an increased prevalence in the population over the age of $40(2,3)$. Apart from flank pain, patients present to ED due to nonspecific reasons, such as fever, nausea, vomiting, uncontrolled hypertension, hematuria, or acute renal failure. This may delay the diagnosis of the disease and increase the risk of possible complications and renal failure (4). Clinical presentation similar to acute pyelonephritis, especially UL, and other diseases that constitute acute abdominal clinics, may cause delays in the diagnosis of infarction (5). The most important step in diagnosis is to consider RI disease because there are no specific laboratory parameters. Contrast-enhanced abdominal computed tomography (CT) is the preferred imaging method to evaluate a variety of acute abdominal conditions. The gold standard method for the diagnosis of RI is contrast-enhanced abdominal CT because it is easy to apply and non-invasive $(6,7)$. Demonstration of a wedge-shaped hypodense lesion in the peripheral area is diagnostic. In addition, elevated white blood cell (WBC), C-reactive protein (CRP), lactate dehydrogenase (LDH), decreased glomerular filtration rate (GFR), and increased creatine (Cr) in patients may help us. $\mathrm{LDH}$ is an indicator of cell necrosis, and its high levels have been shown as a promising marker for predicting the renal prognosis $(3,4,6-8)$. 
This study aimed to investigate whether laboratory parameters could be used to make a distinction between patients with RI, which is a rare clinic in $\mathrm{ED}$, and patients with UL, which is one of the main reasons for flank pain presentations.

\section{MATERIAL AND METHOD}

The study, which was designed retrospectively, was approved by the The study was approved by the Balıkesir University Faculty of Medicine Clinical Research Ethics Committee(Date:23.12.2020, Decision No:2020/238). All procedures were carried out in accordance with the ethical rules and the principles of the Declaration of Helsinki. This study was conducted with the documentation of patients who were aged 18 or older and who presented to University Faculty of Medicine Emergency Department with flank pain between February 01, 2019 and November 01,2020 . Patients younger than 18 years of age were not included in the study. According to the documentation, it was determined that 181 patients presented to the ED with flank pain complaints. However, some of them were excluded from the study because 18 had trauma, 58 had an infection in their complete urinalysis, and 32 had incomplete examinations. A total of 73 patients were included in the study. Of the laboratory values of the patients, WBC, neutrophil, lymphocyte, platelet (Plt), mean platelet volume (MPV), Urea, creatinine (Cr) and LDH were recorded. Neutrophil lymphocyte ratio (NLR) and platelet lymphocyte ratio (PLR) were calculated. The final diagnosis of all patients was confirmed by contrastenhanced abdominal computed tomography results. Our data were collected by trained researchers.

\section{Statistical Analysis}

Shapiro-Wilk test was used to test the normality of variables. Continuous variables were presented as mean \pm standard deviation values for normally distributed variables and with median (1st quartile-3rd quartile) values for non-normal variables. Mann-Whitney U-test was used for comparing two independent groups. Categorical variables were expressed by counts and percentages. Comparisons between the groups were performed with Fisher's exact chi-square test for categorical variables. Risk factors were also evaluated with binary logistic regression analysis. Receiver operating characteristics (ROC) curve analysis was performed to evaluate and compare the performances of diagnostic markers. Youden J index was used to obtain optimal cutoff value, and related sensitivity, specificity, positive predictive, and negative predictive values were given. The significance level was taken as $\alpha=0.05$. The statistical analyses were performed with IBM SPSS Statistics version 22.0 (IBM Corp., USA) and MedCalc version 12.3.0.0 software packages.

\section{RESULTS}

The study included 73 patients, $8(10.96 \%)$ of whom had renal infarct (RI) and $65(89.04 \%)$ of whom had urolithiasis (UL). The mean age of the study sample was $45.45 \pm 14.37$ years. Of the 73 patients, $44(60.27 \%)$ were male, and 29 (39.73\%) were female. Among the RI group, complete resolution of the thrombus was observed in 3 $(37.50 \%)$ patients, and partial resolution of the thrombus was observed in $5(62.50 \%)$ patients.

There was a significant difference between RI and UL groups in terms of LDH $(\mathrm{p}<0.001)$ and PLR $(\mathrm{p}=0.045)$. The values were significantly higher in the RI group compared to the UL group. There was no significant difference between the two groups in terms of the other variables (Table 1).

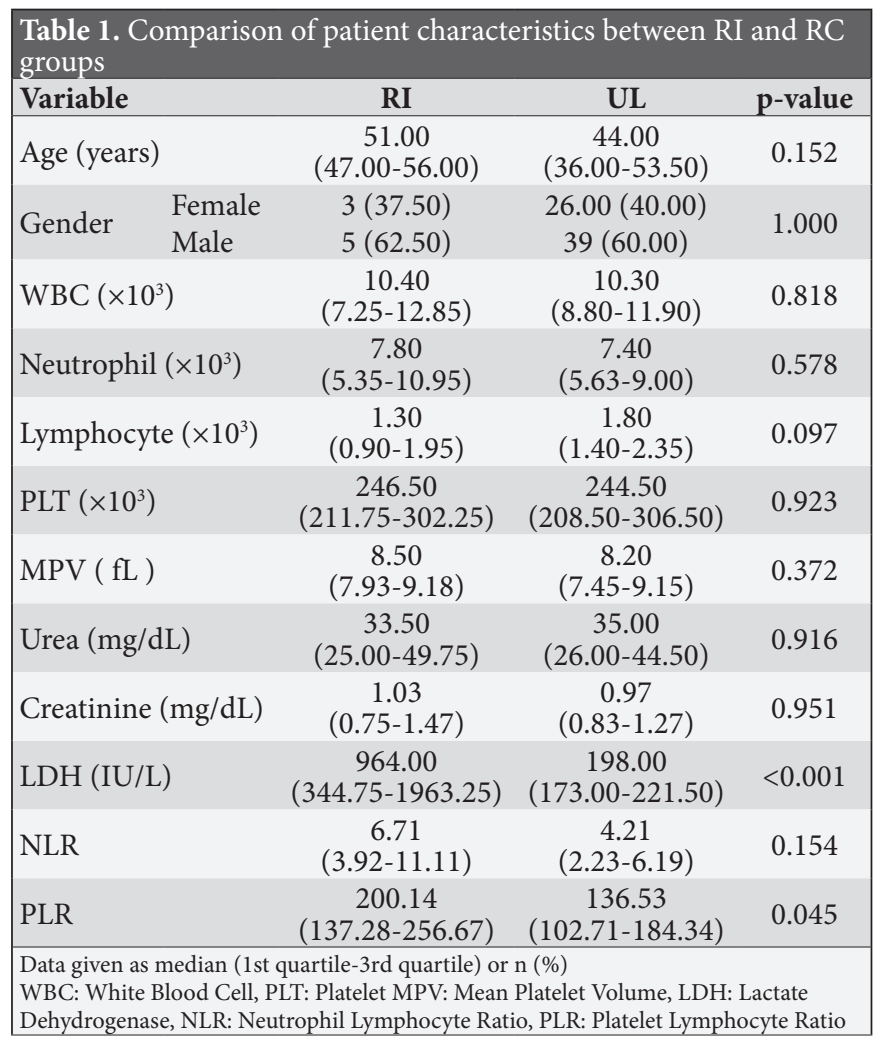

We performed ROC curve analyses to evaluate the diagnostic performances of WBC, neutrophil, lymphocyte, Plt, MPV, urea, Cr, LDH, NLR, and PLR in discriminating patients who had RI and UL. Significant diagnostic performances were obtained for LDH (AUC $=0.983, p<0.001)$ and PLR $(\mathrm{AUC}=0.719$, $\mathrm{p}=0.015)$. Optimal cutoff values were obtained according to the Youden $\mathrm{J}$ index, and corresponding sensitivity and specificity values were given. The WBC $(\mathrm{p}=0.848)$, neutrophil $(\mathrm{p}=0.632)$, lymphocyte $(\mathrm{p}=0.083)$, Plt $(\mathrm{p}=0.912), \operatorname{MPV}(\mathrm{p}=0.271)$, urea $(\mathrm{p}=0.933), \mathrm{Cr}(\mathrm{p}=0.960)$, and NLR $(p=0.175)$ values did not yield significant diagnostic performance in distinguishing between RI and UL groups (Table 2, Figure 1). 


\begin{tabular}{|lcc|}
\hline \multicolumn{4}{|l|}{ Table 2. Diagnostic accuracy measures for LDH and PLR } \\
\hline Diagnostic accuracy measure & LDH & PLR \\
\hline AUC & 0.983 & 0.719 \\
p-value & $<0.001$ & 0.015 \\
Cutoff value & $>259$ & $>225$ \\
Youden-J index & 0.892 & 0.391 \\
Sensitivity (95\% CI) & 100.00 & 50.00 \\
& $(63.10-100.00)$ & $(15.70-84.30)$ \\
Specificity (95\% CI) & 89.23 & 89.06 \\
& $(79.10-95.60)$ & $(78.80-95.50)$ \\
PPV (95\% CI) & 53.3 & 36.4 \\
& $(25.70-79.50)$ & $(10.90-69.20)$ \\
NPV (95\% CI) & 100.0 & 93.4 \\
& $(93.80-100.00)$ & $(84.10-98.20)$ \\
\hline AUC: Area under the curve, CI: Confidence interval, PPV: Positive predictive value, NPV: \\
Negative predictive value, LDH: Lactate dehydrogenase, PLR: Platelet lymphocyte ratio \\
\hline
\end{tabular}

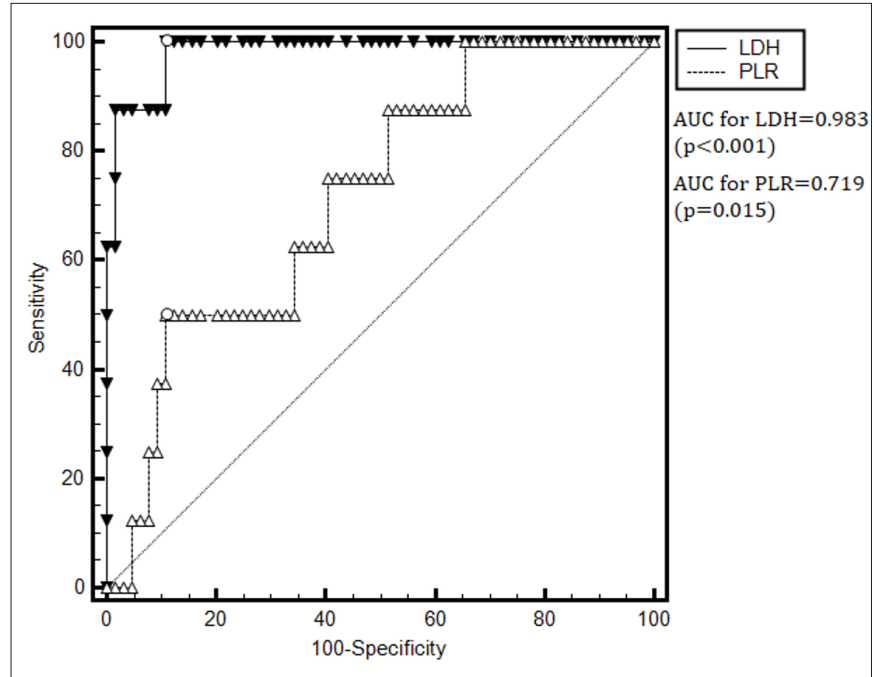

Figure 1. ROC curve for LDH and PLR

We grouped patients according to their LDH and PLR values for the cutoff point obtained from the ROC curve analysis. In the RI group, 8 of the 8 patients (100\%) had $\mathrm{LDH}$ values of $>259 \mathrm{IU} / \mathrm{L}$, and 58 of the 65 patients $(89.23 \%)$ in the UL group had LDH values of $\leq 259$ IU/L

(Figure 2). Also, in the RI group, 4 of the 8 patients (50\%) had PLR values of $>225$, and 57 of the 64 patients $(89.06 \%)$ in the UL group had PLR values of $\leq 225$.

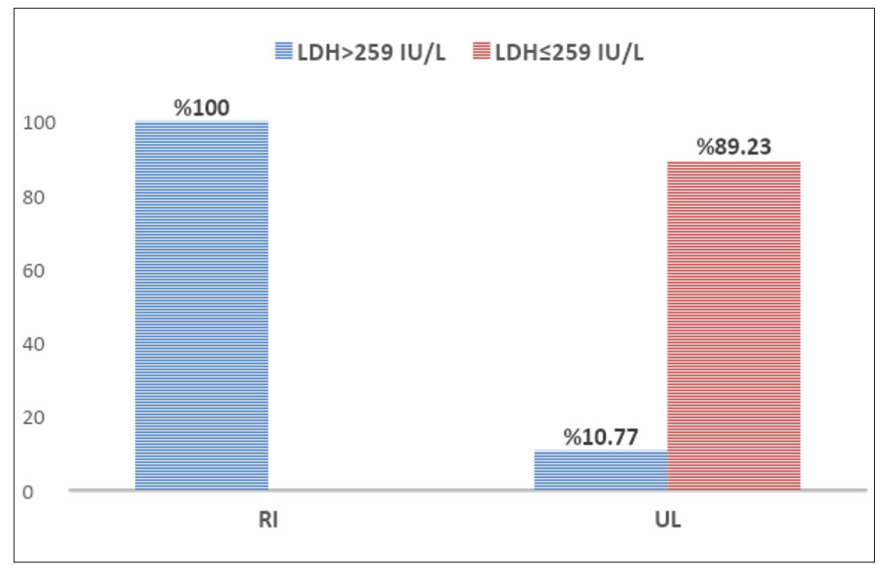

Figure 2. Percentages of RI and UL groups for $\mathrm{LDH}>259$ and $\mathrm{LDH} \leq 259$ patients

\section{DISCUSSION}

The mean age of ED presentations with flank pain concentrates in the 4th decade of life. According to the results of the study involving 400 patients which was conducted by Taşlıdere (9) (2018), the mean age of the patients was $38.22 \pm 14.5$ years (1). Similarly, RI is more common in patients over the age of 40 (2). In our study, the mean age of all patients presenting with flank pain was $45.45 \pm 14.37$, and the median age of patients with RI was 51 years, which is consistent with the literature.

A significant increase in WBC and LDH levels has been found as the most common laboratory parameter in RI patients (10). According to the results of a review study consisting of 102 patients, which was conducted by Antopolsky et al. (10), LDH levels were found to be significantly higher in $95 \%$ of patients with RI, and WBC was shown to increase, as well (11). According to the results of a study that involved 121 patients with RI and was conducted by Eren et al. (2), LDH was found high $(696 \pm 93 \mathrm{IU} / \mathrm{l})$. In the literature, the RI dimension and LDH level were determined in direct proportion, and it was specified as high (6-11). Similarly, LDH was found high in our study, too. WBC and CRP are directly proportional to decreased acute stage renal function (6). According to the study of Silverberg et al. (5) (2016), WBC was found to be high in $67 \%$ of the patients with RI, and CRP was high in $72 \%$ of them. In our study, we found that the WBC count was not increased in both the RI group and the UL group. We think that this was because patients with flank pain due to infection were not included in the study. Besides, the $\mathrm{Cr}$ values of our patients were within the normal range, which may explain the absence of an increase in WBC and CRP levels.

NLR and PLR increase secondary to inflammation, and it is stated in the literature that it can be employed as an alternative to WBC (12). As a result of a 30-day mortality survey on 688 patients who presented to ED with abdominal pain, it was reported that the increase in NLR and PLR was directly proportional to mortality (13). When the rate of NLR and PLR of patients with UL and acute appendicitis (AA) was compared, it was found to be significantly higher in the AA group (14). However, there are no studies in the literature regarding the correlation between PLR and NLR between RI and UL groups, and the results of our study are the first in this regard. While we did not find a significant change in the NLR rate, we found the PLR rate high, and we think it can be used for diagnosis.

In studies conducted on patients with $\mathrm{UL}, \mathrm{Cr}$ values $(0.7 \pm 0.56 \mathrm{mg} / \mathrm{dL}-0.96 \pm 0.19 \mathrm{mg} / \mathrm{dL})$ were found to be normal (9-15). According to the study of Eren et al. (2) (2018), the mean Cr value of 121 patients was 
reported to be minimally high as $1.5 \pm 0.1 \mathrm{mg} / \mathrm{dl}$, and ABY was determined in $35 \%$ of the patients. According to the study of Antopolsky et al. (10) (2012), a moderate increase was found in Cr levels of $43.96 \%$ of the patients. According to our results, no significant increase was observed in the $\mathrm{Cr}$ value, and no difference was found between the groups, either. We think this may have been because the diagnoses of the patients had been made in the emergency department before the kidney functions of the patients were impaired and the patients may have increased their oral hydration for urolithiasis.

\section{CONCLUSION}

Urolithiasis-focused radiological scanning alone in patients who present to ED with flank pain may cause the diagnosis of RI to be overlooked. Therefore, complications, morbidity, and mortality will increase in these patients. We argue that the $\mathrm{LDH}$ value should definitely be studied in patients who present to ED with flank pain, additionally, PLR rate should be calculated, and that in case of detection at high levels, RI diagnosis should be ruled out.

Limitations: Our study was carried out in a single center. The majority of the articles on RI are case reports, and similarly, we have some limitations in terms of the number of patients. The reason why our study group included a small number of patients is that RI is a very rare disease.

\section{ETHICAL DECLARATIONS}

Ethics Committee Approval: The study was approved by the Balıkesir University Faculty of Medicine Clinical Research Ethics Committee (Date: 23.12.2020, Decision No: 2020/238).

Informed Consent: Because the study was designed retrospectively, no written informed consent form was obtained from patients.

Referee Evaluation Process: Externally peer-reviewed.

Conflict of Interest Statement: The authors have no conflicts of interest to declare.

Financial Disclosure: The authors declared that this study has received no financial support.

Acknowledgements: We thank to our colleagues, Department of Emergency and University Hospital who provided insight and expertise that greatly assisted the research.

Author Contributions: All of the authors declare that they have all participated in the design, execution, and analysis of the paper, and that they have approved the final version.

\section{REFERENCES}

1. Çalık M, Büyükcam F, Zengin Y, Afacan MA, Odabaş Ö. Acil servise yan ağrısı ile başvuru nedenleri ve ürolitiyaziste klinik değerlendirme. Yeni Üroloji Derg 2014; 9: 28-33

2. Eren N, Gungor O, Kocyigit I, et al. Acute renal infarction in Turkey: a review of 121 cases. Int Urol Nephrol 2018; 50: 2067-72

3. Bourgault M, Grimbert P, Verret C, et al. Acute renal infarction: a case series. Clin J Am Soc Nephrol 2013; 8: 392-8.

4. Silverberg D, Menes T, Rimon U, Salomon O, Halak M. Acute renal artery occlusion: Presentation, treatment, and outcome. J Vasc Surg 2016; 64: 1026-32.

5. Mesiano P, Rollino C, Beltrame G, et al. Acute renal infarction: a single center experience. J Nephrol 2016; 30: 103-7.

6. Kagaya S, Yoshie O, Fukami H, et al. Renal infarct volume and renal function decline in acute and chronic phases. Clin Exp Nephrol 2017; 21: 1030-4.

7. Markabawi D, Singh-Gambhir H. Acute renal infarction: a diagnostic challenge. Am J Emerg Med 2018; 36: 1325.e1-1325.e2

8. Bae EJ, Hwang K, Jang $\mathrm{HN}$, et al. A retrospective study of short- and long-term effects on renal function after acute renal infarction. Renal Failure, 2014; 36: 1385-9.

9. Taşlıdere B. Acil Serviste Üriner Sistem Taş Hastalığ Analizi, 1.Gastrointestinal Araştırma Kongresi, Malatya, Turkey, Pp.1, 2018

10. Antopolsky M, Simanovsky N, Stalnikowicz R, Salameh S, Hiller $\mathrm{N}$. Renal infarction in the ED: 10-year experience and review of the literatüre. Am J Emerg Med 2012; 30: 1055-60.

11. Domanovits H, Paulis M, Nikfardjam M, et al. Acute renal infarction: clinical characteristics of 17 patients. Medicine (Baltimore) 1999; 78: 386-94

12.Suppiah A, Malde D, Arab T, et al. The prognostic value of the neutrophil-lymphocyte ratio (NLR) in acutepancreatitis: identification of an optimal NLR. J Gastrointest Surg 2013; 17: 675-81.

13. Öncül MV, Dağar S, Emektar E, Çorbacıoğlu ŞK, Aytar H, Çevik Y. Effects of systemic inflammatory parameters on mortality in elderly patients admitted to emergency department with abdominal pain. İstanbul Med J 2019; 20: 125-9.

14. Chen L, Zhang Y. Comparison of the diagnostic values of leukocytes, neutrophils, neutrophil-to-lymphocyte ratio and platelet-to-lymphocyte ratio in distinguishing between acute appendicitis and right ureterolithiasis. Clin Lab 2020; 66.

15.Günaydın GP, Doğan NÖ, Çevik Y, Korkmaz H, Savrun A, Çıkrıkçı G. Evaluation of patients with renal colic that present to an emergency department during the month of Ramadan. JAEM 2013; 12: 24-6. 